\title{
Evaluation of Helicobacter pylori infection in patients with common migraine headache
}

Morteza Hosseinzadeh ${ }^{1}$, Afra Khosravi ${ }^{1}$, Kourosh Saki², Reza Ranjbar ${ }^{3}$

${ }^{1}$ Department of Immunology, Faculty of Medicine, Ilam University of Medical Sciences, Ilam, Iran

2Department of Psychology, Ilam University of Medical Sciences, Ilam, Iran ${ }^{3}$ Molecular Biology Research Center, Baqhiatallah University of Medical Sciences, Tehran, Iran

Submitted: 2 February 2011

Accepted: 16 April 2011

Arch Med Sci 2011; 7, 5: 844-849

DOI: $10.5114 /$ aoms.2011.25560

Copyright ๑ 2011 Termedia \& Banach

\section{Abstract}

Introduction: Migraine can cause headache in different communities so that $12-15 \%$ are suffering worldwide. Recently the relationship between infectious diseases such as Helicobacter pylori infection and migraine headache has been the focus of many studies. The current study was designed to evaluate IgG and IgM antibodies to $\mathrm{H}$. pylori in patients suffering from migraine headaches.

Material and methods: Patients who had diagnostic criteria for migraine were chosen as cases compared to some healthy individuals as the control group amongst which immunoglobulin G (IgG), immunoglobulin $M$ (IgM), age, job, gastro-intestinal (GI) disorders, history of migraine, special meals, medications, sleeping disorders, stress, environmental factors etc were analysed.

Results: The prevalence of disease was $38.6 \%$. Household women had the highest prevalence (40\%). Among them menstruation was related to high prevalence of migraine. $75.6 \%$ of patients had gastrointestinal disorders of which the gastric reflux was the most important sign (47.1\%). The mean optical density (OD) value of IgG and IgM antibody to $H$. pylori was $60.08 \pm 7.7$ and $32.1 \pm 8.7$ for the case group, $21.82 \pm 6.2$ and $17.6 \pm 9.4$ for the control group, respectively. Conclusions: There was a significant difference in mean OD value of both antibodies to $H$. pylori amongst the case and control groups. As a result, active $H$. pylori infection is strongly related to the outbreak and severity of migraine headaches, and $H$. pylori treatment reduces migraine headaches significantly. Hopefully, the definite treatment and eradication of this infection can cure or reduce the severity and course of migraine headaches significantly if not totally.

Key words: migraine, Helicobacter pylori, antibody titre, headache.

\section{Introduction}

Migraine is one of the most common types of headache worldwide, affecting $12-15 \%$ of the world population and also the main headache for which patients refer to specialist nerve clinics. The headache can be mild to severe and might have one or all symptoms such as photophobia, throbbing, nausea and vomiting. The pain severity depends on the activity and number of its outbreaks [1-6]. Migraine is divided into 4 categories: migraine with aura (classic migraine), migraine without aura (common), other types of migraine, and cluster headache [5-7]. Different types of migraine have been proposed in most resources including ophthalmological migraine, proximate migraine, migraine with complication, children's

\author{
Corresponding author: \\ Afra Khosravi MSc, PhD \\ Department of Immunology \\ Faculty of Medicine \\ Ilam University \\ of Medical Sciences \\ Ilam, Iran \\ Phone: $+98-841-2227140$ \\ Fax: $+98-841-227140$ \\ E-mail: \\ afrakhosravi@yahoo.co.uk
}


migraine and abdominal migraine $[5,6]$. When differential diagnosis of migraine is the case, all types of headaches should be considered, such as acute superlative sinusitis, glaucoma, bacterial meningitis, subarachnoid haemorrhage, pheochromocytoma, abnormalities of brain vessels, high blood pressure after stress headaches, cluster headaches and brain tumours [5]. The pathogenesis of headache is divided into three phases: the first phase is pain at the back of the brainstem; the second is vasomotor activity causing contraction or expansion of arteries inside and outside the brain; and the third phase is activation of medullar nerve cells of triple core (Caudalis) and consequently neuropeptide release of vasomotor at the end of the triple nerve vessels $[5,6]$. It also seems that serotonin has a significant role in the creation of migraine headache. Simulating serotonergic cells cause migraine headaches, increasing cerebral blood flow, and also reserpine, which is a serotonin evacuator, is considered as a cause of pain in migraine [7, 12].

Many factors such as genetics, food and medications, sleep disorders, stress, environmental factors such as noise, light, different smells and humidity, work-related factors, menstruation, severe trauma, alcohol, etc, have been recognized as facilitating factors and the cause of migraine headaches so far [5]. Recently the role of infectious diseases, the immune responses, and also the impact of digestive system disorders on migraine headaches have been noted.

This is a flagellated bacteria from the Spirillaceae family of which 11 serotypes in the stomachs of other mammals are recognized, though only the $H$. pylori type has been seen in humans. Some researchers have studied the role of this bacterium in creating acute and chronic gastritis, and gastrointestinal lesions, adenocarcinoma and lymphoma of the stomach [14].

Due to the fact that this bacterium can cause the secretion of serotonin from platelets, releasing PAF, it can play a role in creating migraine headaches by increased secretion of serotonin. However, new research that started in 2002 focused on the role of $H$. pylori activity in creating migraine headaches. As the prevalence of $H$. pylori infection amongst adults living in developed and developing countries is relatively high $(40-50 \%$ and $80 \%$ respectively) and because there is a high proportion of children infected by $\mathrm{H}$. pylori, consequently increasing the prevalence of migraine headaches, the current study was designed to evaluate migraine headaches and their relationship with the antibody titre to $H$. pylori infections.

\section{Material and methods}

This is a case-control study analysing 70 patients with definite diagnosis of migraine including 24 men
(33.4\%), and 46 women (76.5\%), with age ranging from 17 to 52 years referring to private clinics in Ilam. The diagnostic criteria of migraine headache used in the current study were according to the International Headache Society including unilateral pain (affecting one half of the head) and pulsating in nature and lasting from $4 \mathrm{~h}$ to $72 \mathrm{~h}$, having some symptoms including nausea, vomiting, photophobia and phonophobia, where the symptoms are aggravated by routine activity.

Also 70 healthy people without any history of migraine were randomly selected as the control group. Required information including age, sex, gastro-intestinal (GI) disorder, job, history of migraine in family, blood groups, diet, degree of headache and sleep disorder was obtained from all the people in the case and control groups by interviewing and completing a questionnaire. The antibody (immunoglobulin G - IgG, immunoglobulin $M-\lg M)$ titre of all individuals in the case and control groups against $H$. pylori infection was assessed by Enzyme Linked Immunosorbent Assay (ELISA). Data are expressed as mean values \pm standard deviation (SD). The one-sample Kolmogorov-Smirnov test was used to check the normality assumption of the age, and continuousness of variables. $\chi^{2}$ test was used to compare nominal and ordinal variables in case and control groups. Values of $p<0.05$ were considered as significant.

This study was presented to the ethical committee of Ilam University of Medical Sciences and was approved by this committee. Written informed consent was provided by all participants.

\section{Results}

The frequency of blood groups $A, B, O$ and $A B$ amongst individuals of case and control groups was $35.7 \%, 9.9 \%, 50 \%$ and $4.3 \%$, respectively (Tables II, III) while $58.5 \%$ were Rh positive. Group O was the most prevalent blood group among patients with migraine. Housewives with the highest prevalence of migraine headaches were in the first place, followed by students in the second and employers in the third place (Table III). About $75.7 \%$ of those patients who had migraine suffered from Gl

Table I. Comparison of age, IgM and IgG in control and migraine groups

\begin{tabular}{|lccc|}
\hline Variables & $\begin{array}{c}\text { Healthy control } \\
(n=70) \\
\text { Mean } \pm \text { SD }\end{array}$ & $\begin{array}{c}\text { Migraine } \\
(n=70) \\
\text { Mean } \pm \text { SD }\end{array}$ & Value of $p$ \\
\hline Age [years] & $35 \pm 9.6$ & $31.5 \pm 8.1$ & 0.12 \\
\hline IgM & $17.6 \pm 9.4$ & $32.1 \pm 8.7$ & 0.03 \\
\hline IgG & $21.82 \pm 6.2$ & $60.08 \pm 7.7$ & 0.04 \\
\hline
\end{tabular}

IgM - immunoglobulin $M, \lg G$ - immunoglobulin $G$ 
Table II. Frequency of each studied variable in migraine group

\begin{tabular}{|lcc|}
\hline Variables & $\begin{array}{c}\text { Highest } \\
\text { frequency }\end{array}$ & Percent \\
\hline Blood group (ABO) & Group O & 50 \\
\hline Blood group (RH) & + & 58.5 \\
\hline Occupation & $\begin{array}{c}\text { Household } \\
\text { women }\end{array}$ & 40 \\
\hline Literacy & Diploma & 30 \\
\hline Gl disorders & + & 75.5 \\
\hline Gl disorder types & Reflux & 47.1 \\
\hline History of migraine in family & + & 54.3 \\
\hline Cause of headache & Stress & 21.4 \\
\hline Origin of headache & Frontal & 55.7 \\
\hline Sleep disorder & Insomnia & 38.6 \\
\hline Special foods & Cheese & 17.1 \\
\hline Impact of stress on migraine & + & 71.4 \\
\hline Impact of noise on migraine & + & 80 \\
\hline Impact of intense light on migraine & + & 51.9 \\
\hline Impact of head trauma on migraine & + & 52.9 \\
\hline Impact of menstruation on migraine & + & 69.5 \\
\hline Loss of appetite and migraine & + & 97.1 \\
\hline Prevalence of anxiety following & + & 91.4 \\
\hline headache & + & \\
\hline Eye pain and migraine & + & + \\
\hline
\end{tabular}

Table III. Comparison of job, sex and environmental parameters in control and migraine groups

\begin{tabular}{|lccc|}
\hline \begin{tabular}{l} 
Parameters \\
Job, $n(\%):$ \\
\hline Household \\
$(n=32)$
\end{tabular} & $\begin{array}{c}\text { Migraine } \\
(n=38)\end{array}$ & Value of $p$ \\
\hline Employee & $4(50)$ & $4(50)$ & 0.96 \\
\hline Other & $16(44)$ & $20(55.6)$ & \\
\hline Sex: & & & \\
\hline Male & $9(37.5)$ & $15(62.5)$ & 0.32 \\
\hline Female & $23(50)$ & $23(50)$ & \\
\hline Chocolate & $1(20)$ & $4(80)$ & 0.34 \\
\hline Spicy foods & $6(75)$ & $2(25)$ & 0.33 \\
\hline Cheese & $4(33)$ & $8(66)$ & 0.41 \\
\hline
\end{tabular}

disorders, too. The GI disorders were mostly reflux (47.1\%), gastric ulcer (17.1\%), and gastritis (4.3\%). It was demonstrated that $54.3 \%$ of migraine patients had history of migraine in their family, mostly in their mothers $(20 \%)$ or their brothers and sisters (12.9\%).

About $48.6 \%$ of patients mentioned a special relationship between the outbreak and the severity of headache on one side and using a special diet on the other side, although the strongest correlation was found for cheese (17.1\%), fatty foods (12.9\%), and spices (11.4\%). Almost $71.4 \%$ of patients believed that stress can stimulate the migraine, and 91.4\% mentioned that anxiety appeared after the headache. It can also be mentioned that $72.9 \%$ of patients had sleep disorders, amongst which insomnia had the highest prevalence (38.6\%). About $80 \%$ of the patients mentioned a relationship between noise and migraine and $31.4 \%$ remembered a relationship between warm weather and the headache. Also $51.9 \%$ showed a correlation between light and the headaches and $38.6 \%$ had experienced a relationship between the outbreak of headache and different smelling. More than $52.9 \%$ mentioned a relationship between the sudden attack and outbreak of headache, and $32.9 \%$ were able to differentiate any specific diseases and migraine headaches, particularly digestive disorders as the most prevalent disease. It was demonstrated that $69.5 \%$ of women had a correlation between their menstruation and the headache. Frontal headaches, appetite, anorexia state and stress were also mentioned as other problems related to migraine headache (Tables II, IV). At last in order to determine the IgG and IgM antibodies against $H$. pylori in all case and control subjects, the mean optical density (OD) values for both antibodies were calculated. The mean OD value of IgG and IgM antibodies to H. pylori was $60.08 \pm 7.7$ and $32.1 \pm 8.7$ in the case group, $21.82 \pm 6.2$ and 17.6 \pm 9.4 for the control group, respectively. The prevalence of migraine and the IgG and IgM titre against $H$. pylori were significantly correlated using independent Student's $t$-test $(p \leq 0.048$ and $p \leq 0.03$ respectively).

\section{Discussion}

The results of the current study showed a strong correlation between the migraine headache and H. pylori infection, Gl disorders, blood groups, stress, IgG and IgM titres, social class and some environmental factors. Bigal et al., in a similar study, investigated migraine in 120000 families using an electronic questionnaire analysing some factors such as age, sex, race, monthly income, marital status and living area. They found that the prevalence of migraine in girls was higher than that in boys, especially in those over 12 years, with a significant difference, as was observed by us in the current study. The prevalence of disease in families with higher annual income was less than that in families with lower annual income, probably due to more stress in low-income families [3], which was in line with the finding of the current study. The results of the current study showed a higher frequency of migraine headache in those with 
Table IV. Evaluation of stress and migraine in case and control groups

\begin{tabular}{|c|c|c|c|c|c|}
\hline & & & \multicolumn{2}{|c|}{ Migraine } & \multirow[t]{2}{*}{ Total } \\
\hline & & & No & Yes & \\
\hline \multirow[t]{6}{*}{ Stress } & No & Count & 12 & 8 & 20 \\
\hline & & $\%$ within stress & $60.0 \%$ & $40.0 \%$ & $100.0 \%$ \\
\hline & Yes & Count & 20 & 30 & 50 \\
\hline & & $\%$ within stress & $40.0 \%$ & $60.0 \%$ & $100.0 \%$ \\
\hline & Total & Count & 32 & 38 & 70 \\
\hline & & $\%$ within stress & $45.7 \%$ & $54.3 \%$ & $100 \%$ \\
\hline
\end{tabular}

digestive disorders, as was observed by Meucci et al., who evaluated two studies, one in 378 patients with digestive disorders, and the other on 310 cases in Italy, showing a significant correlation between migraine and digestive disorder in women ( $p<0.006)$, while there was no significant correlation between those parameters in men [12]. This finding was further confirmed by a study conducted by IBS medical centre that emphasized a direct relationship between digestive disorders and strength of the migraine headaches. Also from the results of the studies conducted by Movromichalis et al. (1995) and Pradier et al. (2005), a correlation was observed between migraine and digestive disorder [18, 19].

The current study showed that most migraine patients had some history of migraine in their family, mostly in their mother, brothers and sisters, which is in agreement with the study conducted by Stewart et al. in 532 migraine patients and a homogeneous control group [23]. Gervil et al. demonstrated that genetic factors play an important role in the aetiology of migraine without aura, with a similar pattern in men and women [12]. The study of Russell et al. that was carried out on 378 migraine patients showed that migraine with aura and without aura had different aetiology and it seemed that migraine without aura developed due to a combination of genetic and environmental factors, while migraine with aura was definitely caused and controlled by genetic factors [4], which is somehow in agreement with our results in that the environmental and genetic factors affected the severity of migraine headache.

Olrich et al. evaluated migraine in monozygotic and dizygotic twins, finding that the headaches were more prevalent in monozygotic twins than the dizygotes (34\% and $12 \%$ respectively), with a significant difference, confirming the importance of genetics in outbreak of migraine. Also the risk of recurrent migraine in monozygotes was $50 \%$ while in dizygotes it was only $21 \%$ [19]. Our study showed a relationship between the outbreak and the severity of migraine headaches in patients consuming some special meals such as cheese, and fatty foods. Anthony et al. evaluated the amount of serotonin in plasma of migraine patients. It was revealed that urinary excretion (5HIAA) which is a serotonin catabolism increased during migraine attacks strongly, confirming our results [1].

Wacogne et al. evaluated 141 migraine patients and 109 homogeneous control persons, reporting that stress and anxiety in the case group were significantly more dominant than in the control group, suggesting that stress is one of the main factors for starting and sustainability of migraine attacks [29], as was revealed by us.

Looking at the results of our research, sleep disorders are present in $72.9 \%$ of migraine patients, with the highest frequency reported for insomnia. Miller et al. also showed that sleep disorders were significantly higher in the case group compared to the control group [20]. The study carried out by Pavia et al. on the relationship of headache and sleep disorders on 25 patients with morning and night headaches revealed that migraine and tension headaches were seen in almost half of all sleep disorders [24].

Our study showed that environmental factors such as noise, hot weather, strong light, and different smelling had a serious effect on the outbreak and severity of migraine headache. Many other studies also showed similar patterns, such as the research done by Rabbins et al. [25].

One result of the current study was the high prevalence and severity of migraine attacks at the time of menstruation (69.5\%). Silberstein in a similar study found that more than $60 \%$ of women studied reported a migraine attack at the beginning of menstruation [26]. Also, in another study with the same pattern, migraine attacks with aura in the first 2 days before the beginning of the menstruation, and in the last day of the menstruation, were significantly more frequent than at other times [27]. The difference of IgG and IgM titre between the 2 groups was significant ( $p<0.048$ and $p<0.03$ ), indicating a correlation between the antibody titre and the outbreak of 
migraine headaches. Tunca et al. in their study concluded that $84.6 \%$ of patients whose infection was eradicated, and $75 \%$ of patients who had benefited from classical treatment, had reduced symptoms of headaches and their headaches were cured [27]. In a study done by Gasbarrini et al., on patients in whom destruction of $H$. pylori was done successfully, a significant difference was observed in reducing frequency, intensity and duration of migraine attacks comparing to those with history of recurrent disease [11]. In another study conducted by Budzyński et al., it was recommended that the $H$. pylori eradication therapy may lead to prolonging the hospitalization-free period in patients suffering from recurrent chest pain [31].

Also, in a study done by Gasbarrini et al. in Italy on 225 migraine patients among whom $\mathrm{H}$. pylori eradication therapy was performed, it was demonstrated that during 6 months follow-up a significant reduction in frequency, density and duration of migraine attacks was observed [10]. As a result the active $H$. pylori infection is strongly related to the outbreak and severity of migraine headaches, and $H$. pylori treatment reduces migraine headaches significantly. As the infection of bacteria coincides with the severity of the migraine headache and also with the progressive stage of the migraine symptoms, this infection can be considered as one aetiology of the migraine headache.

Finally, according to the results obtained in this study and similar research, the significant difference existing in IgG and IgM antibody titre against $H$. pylori in case compared to control groups shows the importance of studying active H. pylori infection in migraine patients, and also gives rise to a hope that definite treatment and eradication of this bacterium can cure or reduce the severity and course of migraine headaches caused by this bacterium significantly if not totally [31], more importantly than other immunological properties [32].

\section{References}

1. Anthony M, Mracp MB, Hinter Berger H, James W. Lancet. Plasma serotonin in migraine and stress. Arch Neurol 1987; 16: 544-52.

2. Anthony M. Plasma free fatty acids and prostaglandine E 1 in migraine and stress. J Head Face Pain 2005; 16: 58-63.

3. Bigal ME, Lipton RB, Winner P. Migraine in adolescents: association with socioeconomic status and family history. Neurology 2007; 64: 16-25.

4. Russell BM. Increased familial risk and evidence of genetic factor in migraine. BMJ 1995; 311: 541-4.

5. Deleu D, Hanssens Y, Wotrhing EA. Symptomatic and prophylactic treatment of migraine: a critical reappraisal. Clin Neuropharmacol 1998; 21: 267-79.
6. Diener HC, Kaube H, Limmroh V. A practical guide to the management and prevention of migraine. Drugs 1998; 56: 811-24.

7. Ferrari MD. Migraine. Lancet 1998; 351: 1043-51.

8. Ganong WF. Review of medical physiology. 19th ed., Appleton \& Lange 1999.

9. Gabrielle M, Franceschi F, Fior G, et al. Beneficial effects of Helicobacter pylori eradication on migraine. J Headache Pain 2001; 2: 39-43.

10. Gasbarrini A, De luco A, Fiore G, et al. Beneficial effects of Helicobacter pylori eradication on migraine. Hepatogastroenterology 1998; 45: 765-70.

11. Gasbarrini A, Gabrielli M, Fiore G, et al. Association between Helicobacter pylori cytotoxic type I. A positive strains and migraine with aura. Cephalagia 2001; 120: 561-2.

12. Gervil M, Ulrich V, Kaprio J, Olesen J, Russell MB. The relative role of genetic and environmental factors in migraine without aura. Neurology 1999; 53: 995-9.

13. Johnson KW, Phebus LA, Cohen ML. Serotonin in migraine: theories, animal models and emerging therapies. Prog Drug Res 1998; 51: 219-44.

14. Kallela M, Farkklla M, Saljohmaa O, Fyhrquist F. Endothelin in migraine Patients. Cephalalgia 1998; 18: 329-32.

15. MacGregor EA, Hackshaw A. Prevalence of migraine on each day of the natural menstrual cycle. Neurology 2004; 63: 351-3.

16. Mascia A, Afra J, Schoenen J. Dopamine and migraine: a review of pharmacological, biochemical, neurophysiological and therapeutic data. Cephalalgia 1998; 18: 174-82.

17. Meucci G, Radelli F, Prado A, et al. Increased prevalence of migraine in patients with uninvestigated dyspepsia referred for open-access upper gastrointestinal endoscopy. Endosc J 2005; 37: 622-5.

18. Movromichalis I, Zaramboukast T, Giala MM. Migraine of gastrointestinal origin. Eur J Pediatr 1995; 154: 406-10.

19. Pradier A, Devarsdu Meyne JF. Migraine and digestive disorders. Gastroenterol Clin Biol 2005; 29: 156-61.

20. Miller VA, Palermo TM, Powers SW, Scher MS, Hershey AD. Migraine headaches and sleep disturbances in children. Headache 2003; 43: 362-8.

21. Panconesi A, Sicuteri R. Headache induced by serotonergic agonists - a key to the interpretation of migraine pathogenesis. Cephalalgia 1997; 17: 3-14.

22. Pardo J, Carracedo A, Munoz I, Castillo J, Lema M, Noya M. Genetic markers: association study in migraine. Cephalagia 1995; 15: 200-4.

23. Stewart WF, Bigal ME, Kolodner K, Dowson A, Liberman $J N$, Lipton RB. Familial risk of migraine. Variation by proband age at onset and headache severity. Neurology 2006; 66: 344-8.

24. Pavia T, Batista A, Martins P, Martins A. The relationship between headaches and sleep disturbances. J Head Face Pain 2005; 35: 540-6.

25. Rabbins L. Precipitating factors in migraine. A retrospective review of 494 patients. J Head Face Pain 2005; 341: 214-6.

26. Silberstein SD. Menstrual migraine. J Womens Health Gend Based Med 1999; 8: 919-31.

27. Tunca A, Turkey C, Tekino O, Kargili A, Erbayrak M. Is Helicobacter pylori infection a risk factor for migraine? A case control study. Acta Neurol Belg 2004; 104: 161-4.

28. Ulrich V, Gervil M, Kyvik K, Olesen J, Russell MB. Evidence of a genetic factor in migraine with aura: a population based Danish Twin Study. Ann Neurol 2001; 45: 35-40. 
29. Wacogne C, Lacoste JP, Guilibert E, Hugues F, Le Jeunne C. Stress - anxiety, depression and migraine. Cephalagia 2003; 23: 451-5.

30. Waters WE. Migraine: intelligence, social class, and familial prevalence. Br Med J 1971; 2: 77-81.

31. Budzyński J. The favourable effect of Helicobacter pylori eradication therapy in patients with recurrent angina-like chest pain and non-responsive to proton pump inhibitors - a preliminary study. Arch Med Sci 2011; 7: 73-80.

32. Styliani P, Hatzistilianou M, Kouvatsi A, et al. Tumour necrosis factor gene polymorphisms and migraine in Greek children. Arch Med Sci 2010; 6: 430-7. 